\title{
Classifying the secondary component of the binary star W Aquilae (Research Note)
}

\author{
T. Danilovich ${ }^{1}$, G. Olofsson ${ }^{2}$, J. H. Black ${ }^{1}$, K. Justtanont ${ }^{1}$, and H. Olofsson ${ }^{1}$ \\ ${ }^{1}$ Department of Earth and Space Sciences, Chalmers University of Technology, Onsala Space Observatory, 43992 Onsala, Sweden \\ e-mail: taissa@chalmers.se \\ 2 Department of Astronomy, Stockholm University, AlbaNova University Center, 10691 Stockholm, Sweden
}

Received 19 February 2014 / Accepted 23 December 2014

\section{ABSTRACT}

\begin{abstract}
Aims. The object W Aql is an asymptotic giant branch (AGB) star with a faint companion. By determining more carefully the properties of the companion, we hope to better constrain the properties of the AGB star.

Methods. We present new spectral observations of the binary star W Aql at minimum and maximum brightness and new photometric observations of W Aql at minimum brightness.

Results. The composite spectrum near minimum light is predominantly from the companion at wavelengths $\lambda<6000 \AA$. This spectrum can be classified as F8 to G0, and the brightness of the companion is that of a dwarf star. Therefore, it can be concluded that the companion is a main sequence star. From this, we are able to constrain the mass of the AGB component to $1.04-3 M_{\odot}$ and the mass of the W Aql system to 2.1-4.1 $M_{\odot}$. Our photometric results are broadly consistent with this classification and suggest that the main sequence component suffers from approximately 2 mag of extinction in the $V$ band primarily due to the dust surrounding the AGB component.
\end{abstract}

Key words. stars: atmospheres - binaries: spectroscopic - binaries: visual - stars: individual: W Aquilae

\section{Introduction}

It has long been known that the S-type asymptotic giant branch (AGB) star W Aql has a faint companion. Herbig (1965) noted that at minimum brightness W Aql took on spectral features similar to an F5 or F8 star, thus first identifying it as a spectroscopic binary. High resolution Hubble Space Telescope (HST) images have since shown that the binary pair is separated by 0.'46 (Ramstedt et al. 2011). The object W Aql is a Mira variable with a period of 490 days and an amplitude of approximately 7 mag, ranging from 7.3 to 14.3 mag in the $V$ band. Due to its variable nature, many different distance calculations have been made for W Aql (Danchi et al. 1994; Groenewegen 1998; Tatebe et al. 2006; Ramstedt et al. 2009). At a distance of $400 \mathrm{pc}$ (Whitelock et al. 2008), this separation corresponds to 190 AU.

More recently, Mayer et al. (2013) used aperture photometry to classify the main sequence component of W Aql. They found a stable magnitude of $V \approx 14.8$, which implies an absolute magnitude of $M_{V}=7.1$ for an unreddened star at their adopted distance of $340 \mathrm{pc}$, which would be consistent with a K4 dwarf. This is very different to Herbig's (1965) initial classification.

Previous studies have shown an extended dusty circumstellar envelope (CSE) around W Aql. In Ramstedt et al.'s (2011) observations of polarised visible light, the CSE extends more than $10^{\prime \prime}$ around the primary, which is well beyond the offset between the two binary components. This would certainly cause extinction depending on the alignment of the system. Tatebe et al. (2006) used the UC Berkeley Infrared Spatial Interferometer to resolve the distribution of emitting dust at $11.15 \mu \mathrm{m}$ and found an asymmetry in the dust distribution around the primary on small scales (hundreds of mas), while Ramstedt et al. (2011) find asymmetry on large scales (10 arcsec). Herschel/PACS photometry presented in Mayer et al. (2013) shows a large ( 100 arcsec) dust envelope on the same general shape as Ramstedt et al. (2011) found in the optical but with an bright patch of dust to the east of the star in both the $70 \mu \mathrm{m}$ and $160 \mu \mathrm{m}$ images.

Having a more precise classification of the main sequence companion allows us to better constrain several properties of the W Aql system. The mass of the main sequence component, which scales with spectral type, puts a lower limit on the initial mass of the AGB component; as the AGB component is more evolved, it must have had a larger initial mass. Further, the main sequence component imparts additional energy to the CSE of the AGB component, which could have an effect on the chemistry of the CSE, for example, through additional heating.

With conflicting results for the classification of the W Aql companion ( $\mathrm{F}$ versus $\mathrm{K}$ spectral types), we investigate the binary system to find a more precise classification. We present new spectroscopic and photometric observations made during the minimum phase of the AGB component and new spectroscopic observations taken during the maximum phase of the AGB star for comparison. We present our observations in Sect. 2, perform the spectral analysis in Sect. 3, analyse the photometry in Sect. 4, and discuss the results in Sect. 5.

\section{Observations and data reduction}

Observations were carried out using the Andalucia Faint Object Spectrograph and Camera (ALFOSC) on the Nordic Optical Telescope (NOT) on two nights in May 2012 and March 2013. The spectroscopy was done with a grism with 600 rules/mm, a dispersion of $1.5 \AA$, and the wavelength range $3850-6850 \AA$. The photometric observations were taken using $U B V$ filters of the Bessel photometric system. The observations are summarised in Table 1 . The seeing on the night of our photometric observations 
Table 1. Observations of W Aql.

\begin{tabular}{cccc}
\hline \hline Night of & Type & Configuration & Phase \\
\hline $2012-05-11$ & spectroscopy & 1'.0 0 slit, Grism \#7 & $\max$ \\
$2013-03-29$ & photometry & U_Bes 362_60 & $\min$ \\
$2013-03-29$ & photometry & B_Bes 440_100 & $\min$ \\
$2013-03-29$ & photometry & V_Bes 530_80 & min \\
2013-03-29 & spectroscopy & 1'.0 slit, Grism \#7 & min \\
\hline
\end{tabular}

Notes. The numerical codes for the photometric filters give the central wavelengths followed by the full width at half maximum (FWHM) in $\mathrm{nm}$. Max and min in the phase column refer to the periods of maximum or minimum brightness of $\mathrm{W}$ Aql.

ranged between $0.5-1{ }^{\prime \prime} 1$, so we were unable to resolve the two components of W Aql.

Data reduction was carried out using IRAF (v 2.16). For the spectroscopy, we used a HeNe lamp for the wavelength calibration.

The standard reference star PG1525-071, and two other stars (labelled A and C) in the same field ${ }^{1}$ (Landolt 1992) were used for our photometric calibration. To compensate for the different spectral response functions between the standard $U B V$ system and the ALFOSC system, we applied the corrections as advised in the ALFOSC manual ${ }^{2}$. The integration times were $3 \times 200 \mathrm{~s}$, $20 \times 15 \mathrm{~s}$, and $10 \times 1 \mathrm{~s}$ in $U, B$, and $V$, respectively. The standard stars were observed at air mass $\approx 1.24$ and $\mathrm{W}$ Aql at air mass $\approx 1.66$, and we used the standard extinction $0.46,0.22$, and $0.12 \mathrm{mag} /$ airmass in $U, B$, and $V$, respectively, to compensate for the differential atmospheric extinction. The $U$ filter has small spectral leaks in the red spectral region beyond $7000 \AA^{3}$, and for extremely red sources, like W Aql, there is a risk for contamination. However, a possible red ghost image would be displaced by $2^{\prime \prime} .5$ at the zenith distance of the observations, $\approx 53^{\circ}$, and we can exclude a red ghost that is brighter than $0.5 \%$ of the brightness in the $U$ band.

\section{Spectral analysis}

When the AGB star is at maximum brightness, it completely dominates the spectrum at almost all wavelengths. The only aspect of the spectrum that might possibly show a contribution from the companion is a slight rise in the continuum in the blue from around $4200 \AA$ and bluewards.

When the AGB star is at minimum brightness, however, several features, which are clearly from the companion star, become visible in the blue end of the spectrum. The companion star dominates the spectrum up to around 5800-6000 , redwards of which features that are characteristic of the AGB star again become apparent. Table 2 summarises all the spectral features and the spectrum itself is shown in Fig. 1.

\subsection{Features present only at minimum brightness}

Most of the features unique to the minimum brightness spectrum are found in the blue end, where the companion star dominates. The Balmer series is the most prominent feature with absorption

\footnotetext{
1 http://wWw.not.iac.es/instruments/stancam/photstd/ pg1525.html

2 http://www.not.iac.es/instruments/alfosc/zpmon/

3 See http://www.not.iac.es/instruments/filters/ curves/png/7.png
}

Table 2. Spectral features at different phases.

\begin{tabular}{lccc}
\hline \hline Feature & $\lambda[\AA]$ & Maximum & Minimum \\
\hline $\mathrm{H} \alpha$ & 6563 & emission & emission \\
$\mathrm{H} \beta$ & 4861 & emission & absorption \\
$\mathrm{H} \gamma$ & 4340 & emission & absorption \\
$\mathrm{H} \delta$ & 4102 & $\ldots$ & absorption \\
$\mathrm{CaII} \mathrm{H}(+\mathrm{H} \varepsilon)$ & 3970 & $\ldots$ & absorption \\
$\mathrm{CaII} \mathrm{K}$ & 3934 & $\ldots$ & absorption \\
$\mathrm{Ca} \mathrm{I}$ & 4226 & $\ldots$ & absorption \\
$\mathrm{Fe} \mathrm{I}$ & 4383 & $\ldots$ & absorption \\
$\mathrm{MgI}$ & $\sim 4300$ & $\ldots$ & absorption \\
$\mathrm{G} \mathrm{band}(\mathrm{CH})$ & 5892,5898 & absorption & absorption \\
$\mathrm{Na} \mathrm{D}$ & $4620,4641,5304$ & present & $\ldots$ \\
$\mathrm{ZrO}$ bands & $5562,5718,5724$ & present & $\ldots$ \\
& 5849 & present & $\ldots$ \\
& $6136,6344,6474$ & present & weaker \\
& 6495 & present & weaker \\
YO bands & 5972,6132 & present & weak \\
\hline
\end{tabular}

References. References for wavelengths: Kramida et al. (2013) and Gray et al. (2009).

lines of $\mathrm{H} \beta, \mathrm{H} \gamma$, and $\mathrm{H} \delta$ present, which are absent in the maximum brightness spectrum. There are also strong $\mathrm{Ca}$ II $\mathrm{K}$ and $\mathrm{H}$ absorption features with the $\mathrm{Ca}$ II $\mathrm{H}$ line possibly blended with $\mathrm{H} \varepsilon$. The strengths and shapes of these lines suggest that the companion star is an F star. The absence of H8 and H9 Balmer absorption lines bluewards of the Ca II pair suggests a later classification than F5 such as F8 (Gray et al. 2009).

Several less prominent metal lines are also present. The weak Ca I $4226 \AA$ line suggests an early to mid-F classification, as does the absence of the Fe I $4046 \AA$ line. The Mg I triplet around $5170 \AA$ is not stronger than the nearby $\mathrm{H} \beta$ line, which suggests an early-F classification. The slightly more prominent Fe I $4383 \AA$ line suggests a mid-F classification. The weakness of these metal lines could be due to the star being relatively metal-poor. This would explain the apparent discrepancy between the metal lines and the Balmer series. The strength of the $G$ band due to the $\mathrm{CH}$ molecule, which is comparable to $\mathrm{H} \gamma$, also supports a later classification (Gray et al. 2009).

Overall, the spectral features suggest an F8 or F9 classification for the companion star and do not conclusively rule out G0.

\subsection{Features present only at maximum brightness}

In stark contrast to the minimum brightness spectrum, the Balmer series lines $\mathrm{H} \beta$ and $\mathrm{H} \gamma$ are strongly in emission, indicating shocks in the AGB star. None of the absorption features discussed in Sect. 3.1 are present.

There are strong $\mathrm{ZrO}$ bands throughout the spectrum but only a few generally weaker TiO bands, which suggests a relatively high $\mathrm{C} / \mathrm{O}$ ratio for the $\mathrm{S} \operatorname{star}(\sim 0.98$, see Sect. 5.3). There are also possible blendings of $\mathrm{ZrO}$ with $\mathrm{YO}$ and $\mathrm{LaO}$ (see Table 2).

\subsection{Features present during both phases}

Mostly features at the red end of the spectrum are visible during both phases.

The sodium doublet (Na D) at 5892 and $5898 \AA$ is present for both phases but with a significant difference in shape. At minimum brightness, the absorption is narrower, and the two components of the doublet are clearly distinguished with a small 
T. Danilovich et al.: Classifying the secondary component of the binary star W Aquilae $(R N)$
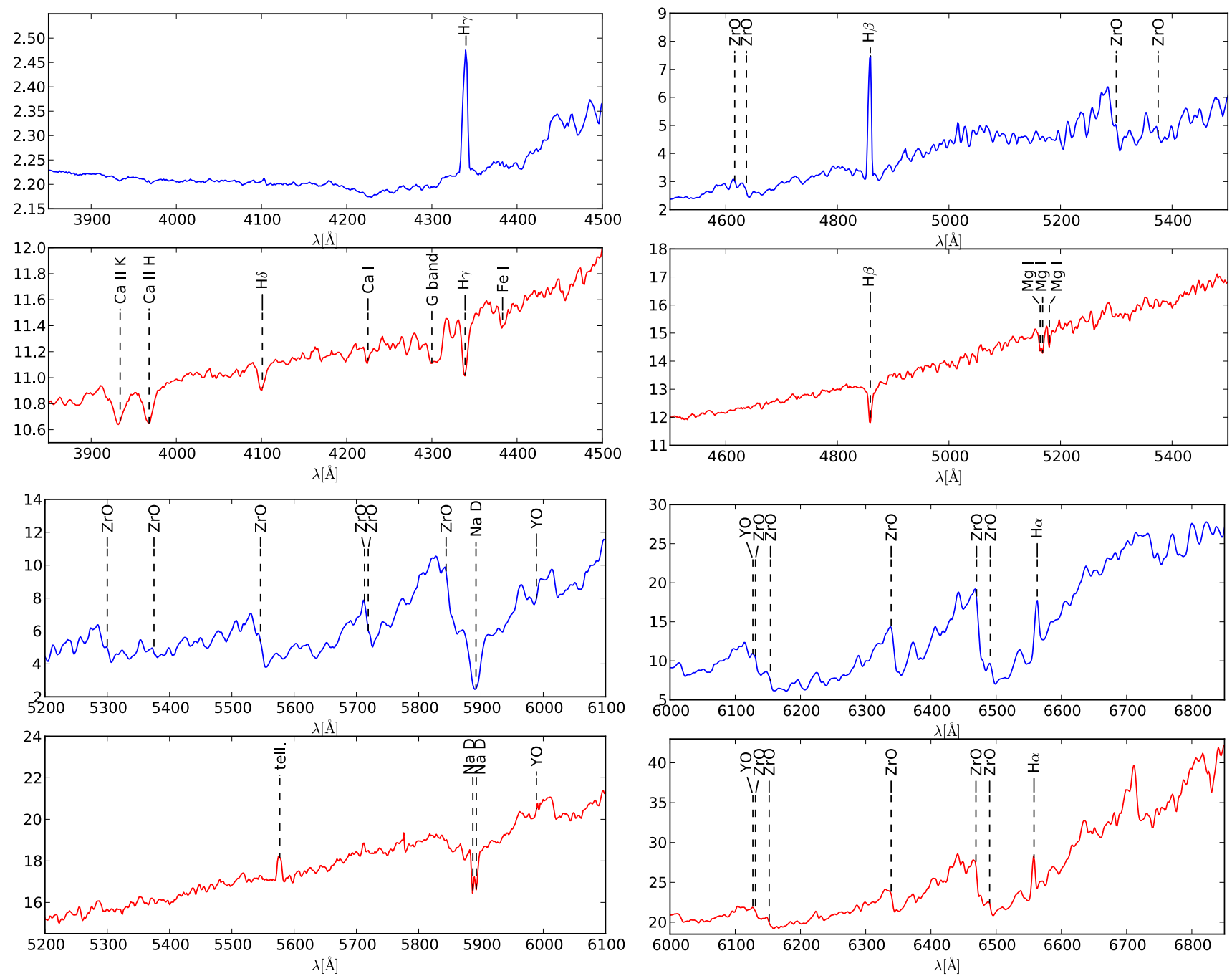

Fig. 1. Observed spectra. The blue curve is the spectrum at maximum brightness and the red curve is the spectrum at minimum brightness. The vertical axes are in arbitrary flux units.

peak between them. At maximum brightness, the Na D absorption is bordered by $\mathrm{ZrO}$ and $\mathrm{YO}$ and is much stronger with broader wings. Although the trough is slightly asymmetric, it is not possible to distinguish the two components. The wings of the $\mathrm{Na} \mathrm{D}$ lines are apparent nearly up to the neighbouring YO band in the maximum brightness spectrum, while there is a clear gap between Na D and the same, albeit weaker, YO band in the minimum brightness spectrum. It is likely that the $\mathrm{Na} \mathrm{D}$ feature comes from the AGB star in both cases, although it is possible there is some contribution from the $\mathrm{F}$ star at minimum brightness.

As for the above-discussed YO band at $5972 \AA$, some redder $\mathrm{ZrO}$ bands in the 6300 to $6500 \AA$ range appear during both phases. The bands are much weaker in the minimum brightness spectrum, which is to be expected.

The final feature common to both phases is the $\mathrm{H} \alpha$ emission line. As with the other Balmer emission lines discussed in Sect. 3.2, it most likely originates in shocks in the AGB star. In both cases, it is located on the side of a broad absorption trough, which is less deep at minimum brightness, making it difficult to compare with the other Balmer lines. Woodsworth (1995) performed a detailed analysis of $\mathrm{H} \alpha$ lines in $\mathrm{S}$ stars at different phases, but we do not have sufficient resolution in our spectrum to compare with his results.
Table 3. Observed photometric results at phase minimum.

\begin{tabular}{ccc}
\hline \hline Band & Flux (mag) & Uncertainty \\
\hline$U$ & 16.01 & 0.03 \\
$B$ & 15.26 & 0.01 \\
$V$ & 14.12 & 0.01 \\
$U-B$ & 0.75 & 0.03 \\
$B-V$ & 1.14 & 0.02 \\
\hline
\end{tabular}

\section{Photometric results}

Our photometric results are summarised in Table 3, which includes the $U-B$ and $B-V$ colours. It should be noted that our photometric results are not exclusively of the fainter companion and include contamination by the AGB star, as the seeing was insufficient to resolve the two components of W Aql.

Based on our observations, we can make some further deductions. If we assume that there is no extinction, which is an unlikely prospect given the dusty AGB envelope (see Danilovich et al. 2014, Fig. 2), we find that the absolute magnitude, assuming a distance of $400 \mathrm{pc}$, is $M_{V, \mathrm{obs}}=6.1 \mathrm{mag}$ in the $V$ band. This would suggest a K1 classification (Gray et al. 2009), similar to what Mayer et al. (2013) found. However, if we instead assume that our spectral classification is accurate, we can use 
canonical values (taken from Cox 2000; Gray et al. 2009) to calculate the extinction. Considering $(B-V)_{0}=0.52$ for an $\mathrm{F} 8 \mathrm{~V}$ star, the excess $E(B-V)=0.62$ is consistent with an extinction of $A_{V}=1.9 \mathrm{mag}$, assuming standard interstellar extinction $\left(R_{V}=3.1\right.$, Draine 2011). If we calculate the extinction based on $M_{V, \mathrm{~F} 8 \mathrm{~V}}=4.0$ instead, we find $A_{V}=M_{V, \mathrm{obs}}-M_{V, \mathrm{~F} 8 \mathrm{~V}}=2.1 \mathrm{mag}$. This could suggest a slight difference in the dust being produced by the AGB star as compared with dust in the interstellar medium (ISM). Another source of uncertainty in the derivation of $A_{V}$ occurs because the $V$ and $B-V$ measurements are not exclusively of the F star but are likely contaminated by the AGB star.

\section{Discussion}

\subsection{Orientation of system}

The AGB component is surrounded by a dusty envelope and the envelope extent is significantly larger than the apparent separation between the AGB star and its companion (Ramstedt et al. 2011; Mayer et al. 2013). The significant extinction we calculate of $A_{V}=2$ suggests that the companion star most likely sits within or behind the AGB star's CSE. Although some of the extinction we see may be interstellar, we believe it is unlikely to account for all the extinction, particularly as no interstellar contamination has been seen in W Aql's molecular emission lines (Danilovich et al. 2014; Ramstedt et al. 2009; Schöier et al. 2013).

\subsection{Properties of the fainter companion}

Mayer et al. (2013) used a luminosity-based classification for the companion. Using HST images, they calculated a K4V classification, which is significantly later than our classification of F8 or F9. We believe the difference arises in the lack of consideration of dust in Mayer et al.'s (2013) analysis.

Spectrally, the absence of the MgH feature at $4780 \AA$ and the $\mathrm{MgH}$ band at $5198 \AA$ in our spectrum (see Fig. 1) counters the $\mathrm{K} 4$ classification, as does the presence of the Balmer absorption lines, the strength of the $\mathrm{Ca}$ II $\mathrm{K}$ and $\mathrm{H}$ absorption lines, and the strength of the $\mathrm{G}$ band, which should be much shallower by the mid-K.

Our result of an F8 to G0 star gives an effective temperature in the range of 6170-5900 K (Gray et al. 2009) and a stellar mass of 1.09-1.04 $M_{\odot}$ (Habets \& Heintze 1981).

\subsection{Properties of the AGB component}

The most prominent spectral features of the AGB component are the multiple $\mathrm{ZrO}$ bands, some of which are still visible in the red end of the spectrum even at the AGB star's minimum brightness. On the other hand, no clear TiO bands are seen in either minimum or maximum spectra. In general, $\mathrm{TiO}$ molecules are less resistant to temperature increases than $\mathrm{ZrO}$ and hence $\mathrm{TiO}$ bands are expected to be weak at maximum brightness and potentially more prominent at minimum brightness (Richardson 1933). Their absence could be due to the companion dominating the spectrum, but this could only be true at the bluer end. The absence of $\mathrm{TiO}$ at the redder end of the spectrum, even at minimum brightness, indicates that the AGB component is more C-like than $\mathrm{M}$-like in terms of the $\mathrm{C} / \mathrm{O}$ abundance index. Following the criteria laid out in Keenan \& Boeshaar (1980), we find C/O 0.98 with the classification of S6/6e (the first 6 being the temperature classification, taken from Keenan \& Boeshaar's (1980) observational results of $\mathrm{W} A q \mathrm{l}$ and the e indicating the presence of emission lines). This is the highest $\mathrm{C} / \mathrm{O}$ ratio before the $\mathrm{SC}$ classification takes over and is broadly consistent with molecular abundances determined in Danilovich et al. (2014).

If we assume that the two components of $\mathrm{W} \mathrm{Aql} \mathrm{formed}$ contemporaneously, before it left the main sequence, the AGB star must have been more massive than the fainter companion. Additionally, the presence of s-process elements and more specifically Tc (Little-Marenin \& Little 1988) constrains the AGB star's mass to less than $\sim 3 M_{\odot}$ (Herwig 2005). This allows us to constrain the total system mass to $2.1-4.1 M_{\odot}$.

\section{Conclusions}

The main conclusions of this paper are as follows:

- Our spectroscopic observations of W Aql suggest that the fainter component of the binary star is an F8 or F9 star due to the strength of the $\mathrm{Ca} \mathrm{II} \mathrm{H}$ and $\mathrm{K}$ lines, the Balmer absorption lines, and the $\mathrm{Ca} \mathrm{I}, \mathrm{Fe} \mathrm{I}$, and $\mathrm{Mg} \mathrm{I}$ lines.

- The strength of the $\mathrm{ZrO}$ bands and lack of $\mathrm{TiO}$ bands in our spectral observations indicates that the AGB component of W Aql is an S6/6e star, which agrees with previous results.

- Our photometric results taken at minimum light are consistent with a star of luminosity class $\mathrm{V}$ at a distance of $400 \mathrm{pc}$ and extinction $A_{V}=2$ mag. Mayer et al. (2013) mistakenly attributed a spectral type of K4 because they neglected the large correction for extinction.

- We are able to constrain the mass of the AGB star to 1.04-3 $M_{\odot}$ based on the classification of the fainter star. This constrains the total system mass to 2.1-4.1 $M_{\odot}$.

Acknowledgements. T.D. and K.J. acknowledge funding from the SNSB. Based on observations made with the Nordic Optical Telescope, operated by the Nordic Optical Telescope Scientific Association at the Observatorio del Roque de los Muchachos, La Palma, Spain, of the Instituto de Astrofisica de Canarias. We acknowledge with thanks the variable star observations from the AAVSO International Database contributed by observers worldwide and used in this research. This research has made use of the International Variable Star Index (VSX) database, operated at AAVSO, Cambridge, Massachusetts, USA.

\section{References}

Cox, A. N. 2000, Allen's Astrophysical Quantities (Berlin: Springer) Danchi, W. C., Bester, M., Degiacomi, C. G., Greenhill, L. J., \& Townes, C. H. 1994, AJ, 107, 1469

Danilovich, T., Bergman, P., Justtanont, K., et al. 2014, A\&A, 569, A76

Draine, B. 2011, Physics of the Interstellar and Intergalactic Medium, Princeton Series in Astrophysics (Princeton University Press)

Gray, R., Corbally, C., \& Burgasser, A. 2009, Stellar Spectral Classification, Princeton Series in Astrophysics (Princeton University Press)

Groenewegen, M. A. T., \& De Jong, T. 1998, A\&A, 337, 797

Habets, G. M. H. J., \& Heintze, J. R. W. 1981, A\&AS, 46, 193

Herbig, G. H. 1965, Veroeffentlichungen der Remeis-Sternwarte zu Bamberg, 27, 164

Herwig, F. 2005, ARA\&A, 43, 435

Keenan, P. C., \& Boeshaar, P. C. 1980, ApJS, 43, 379

Kramida, A., Ralchenko, Yu, Reader, J., and NIST ASD Team, 2013, NIST Atomic Spectra Database (v. 5.1), National Institute of Standards and Technology, Gaithersburg, MD, http://physics.nist.gov/asd Landolt, A. U. 1992, AJ, 104, 340

Little-Marenin, I. R., \& Little, S. J. 1988, ApJ, 333, 305

Mayer, A., Jorissen, A., Kerschbaum, F., et al. 2013, A\&A, 549, A69

Ramstedt, S., Schöier, F. L., \& Olofsson, H. 2009, A\&A, 499, 515

Ramstedt, S., Maercker, M., Olofsson, G., Olofsson, H., \& Schöier, F. L. 2011, A\&A, 531, A148

Richardson, R. S. 1933, ApJ, 78, 354

Schöier, F. L., Ramstedt, S., Olofsson, H., et al. 2013, A\&A, 550, A78

Tatebe, K., Chandler, A. A., Hale, D. D. S., \& Townes, C. H. 2006, ApJ, 652 , 666

Whitelock, P. A., Feast, M. W., \& van Leeuwen, F. 2008, MNRAS, 386, 313

Woodsworth, A. W. 1995, ApJ, 444, 396 